http://dx.doi.org/10.18359/ravi.2199

\title{
Normatividad y estrategias de formación de profesores en tecnologías de la información y la comunicación ${ }^{1}$
}

\author{
Lillyam López de Parra² , Magda Julissa Rojas Bahamón ${ }^{3}$, Lucelly Correa Cruz ${ }^{4}$, Diego Arbeláez Campillo 5 \\ Universidad de la Amazonia, Colombia
}

Recibido, julio 18 de 2016

Concepto evaluación, diciembre 19 de 2016

Aceptado, diciembre 27 de 2016

\author{
Referencia: López de Parra, L.; Rojas Bahamón, M.; Correa \\ Cruz, L. \& Arbeláez Campillo, D. (2017). "Normatividad \\ y estrategias de formación de profesores en tecnologías de \\ la información y la comunicación". Revista Academia y \\ Virtualidad, 10, (1), 79-94
}

\section{Resumen}

El objetivo de este artículo consiste en analizar las normas y estrategias implementadas en el país para la formación de profesores en el uso de las TIC. La metodología se ciñe a la investigación cualitativa de carácter documental; para ello, se revisaron y sistematizaron documentos sobre lineamientos de políticas de formación docente para el uso de las TIC en el ámbito internacional y nacional y, asimismo, las estrategias de formación en TIC. Los resultados señalan que los lineamientos de políticas sobre formación en TIC tienen tres ejes importantes: acceso a las TIC, formación de profesores y configuración de redes. El propósito de la formación en TIC es obtener competitividad, calidad y equidad de la educación. Las estrategias de formación de profesores se han realizado por niveles y privilegiado la técnica de cascada. Las conclusiones destacan que varias de las estrategias de formación implementadas aún no superan la formación instrumentalista de las TIC y no han logrado ahondar en la fundamentación pedagógica, comunicativa e investigativa, tal como se plantea en la normatividad. Por consiguiente, se requiere fundamentar el empleo de las TIC desde la visión crítica y la perspectiva de mediación cultural.

\footnotetext{
${ }^{1}$ Artículo resultado del proyecto de investigación: "Representaciones Sociales sobre formación en Tecnologías de la Información y Comunicación. Profesores Educación Básica Secundaria”, financiado por la Vicerrectoría de Investigaciones de la Universidad de la Amazonia.

${ }^{2}$ Doctora en Educación; Profesora Facultad de Ciencias de la Educación de la Universidad de la Amazonia, Florencia, Caquetá, Colombia; Integrante Grupo de Investigación "Lenguajes, Representaciones y Educación”, Universidad de la Amazonia. mar.lopez@udla.edu.co.

${ }^{3}$ Magíster en Educación; Doctorando en Educación y Cultura Ambiental, Universidad de la Amazonia; Universidad Sur Colombiana; Profesora, Institución Educativa Antonio Ricaurte, Florencia, Caquetá, Colombia; Integrante Grupo de Investigación “Lenguajes, Representaciones y Educación”, Universidad de la Amazonia.mjulissa@iear.edu.co.

${ }^{4}$ Magíster en Educación; Doctorando en Educación y Cultura Ambiental, Universidad de la Amazonia; Universidad Sur Colombiana; Profesora, Facultad de Ciencias Contables, Económicas y Administrativas, Universidad de la Amazonia, Florencia, Caquetá, Colombia. Integrante Grupo de Investigación “Lenguajes, Representaciones y Educación”, Universidad de la Amazonia. 1.correa@udla.edu.co.

${ }^{5}$ Magíster en Educación; Integrante Grupo de Investigación “Lenguajes, Representaciones y Educación”, la Universidad de la Amazonia, Colombia.dfaca@hotmail.com.
} 


\title{
ICTs regulations and strategies for teacher training
}

\begin{abstract}
The aim of this paper is analyzing the regulations and strategies implemented in our country for teachers' training in order to use ICTs. The methodology used was a qualitative documentary research where we checked and systematized several papers about policy guidelines and strategies for teacher training to use ICTs at the local and international levels. Results indicate that training in ICTs policy guidelines have three critical issues: access ICTs, teacher training, and network settings. The purpose of the ICTs training is getting competitiveness, quality and equity of education. Teacher training strategies haven been performed by levels and emphasized the waterfall technique. Conclusions have highlighted that several strategies of training implemented just barely meet instrumental ICTs training and have failed to delve deeper into the educational, communication and research foundation, as the regulations set out. Then it is required to base the ICTs use from a critical vision and a cultural focus.
\end{abstract}

Keywords: information and communications technologies, ICTs regulations, ICTs training strategies, teachers, teacher training.

\section{Normatividade e estratégias de formação de professores nas tecnologias da informação e comunicação}

\section{Resumo}

O objetivo deste artigo tem a ver com a análise das normativas e estratégias implementadas no pais a fim de formar professores no uso das TIC. A metodologia vai ligada à pesquisa qualitativa de caráter documental; para isso se revisaram e sistematizaram documentos sobre lineamentos de políticas de formação docente para o uso das TIC no âmbito internacional e nacional e, assim mesmo, as estratégias de formação em TIC. Os resultados sinalam que os lineamentos de política sobre formação em TIC têm três eixos principais: acesso as TIC, formação de professores e configuração de redes. O propósito da formação em TIC é de obter a competividade, qualidade e equidade na educação. As estratégias da formação de professores têm se realizado por níveis e privilegiado as técnicas de cascata. As conclusões destacam que várias das estratégias de formação implementadas ainda não superam a formação instrumentalista das TIC e não têm logrado afundar na fundamentação pedagógica, comunicativa e pesquisadora, tal qual vem proposto na normatividade. Em consequência, precisa-se de fundamentar o emprego das TIC desde a visão crítica e a perspectiva da mediação cultural.

Palavras chave: tecnologias da informação e a comunicação; normatividade TIC; estratégias em formação de TIC; professores; formação docente. 


\section{Introducción}

La inserción de las tecnologías de la información y la comunicación (TIC) ${ }^{6}$, se puede decir, constituyen un acontecimiento en el ámbito educativo; según Krüger (2006), tienen la disposición para la innovación permanente del conocimiento; en este sentido, las TIC irrumpen y permean todas las esferas de la sociedad.

Para implementar el empleo de lasTIC en las prácticas educativas se requirió su regulación mediante la expedición y aplicación de normas. Según Pérez (1996), “[...] desde una perspectiva psicosocial, las normas constituyen un elemento regulador de la conducta de los individuos en la sociedad, en la medida que determinan lo que hay que hacer o no" (Ibíd, p. 535). Siguiendo al autor, las normas tienen un enunciado o principio valorativo y, en general, "son medios o instrumentos para conseguir determinadas metas" (Ibíd, p. 535); es decir, tienen, por una parte, la función de regulación $\mathrm{y}$, por el otro, la instrumental, para obtener resultados. Para Popkewitz (1994),

[...] la producción de reglas y normas a través de las prácticas sociales forma parte de los procesos de regulación. En determinado nivel, las normas públicas sobre escolarización pueden ser útiles como guías que orienten a los profesores y como puntos de referencia en el debate entre los elementos constituyentes de la escolarización respecto a lo más apropiado y digno de atención (p. 151).

Asimismo, en el marco de las reformas en el contexto educativo, denominadas de la competitividad (Carnoy, 2001) se encuentran las relacionadas con la implementación de las TIC. "Las reformas basadas en la competitividad, ante todo pretenden aumentar la productividad económica mejorando la calidad de la mano de obra" (p. 101). De ahí, la necesidad de buscar la pertinencia de la enseñanza en relación con el mundo del trabajo, para incrementar la producción.

En consonancia, otras demandas son la ampliación de la infraestructura de comunicaciones y elevar la calidad de la formación tecnológica de los profesores y comunidad en general, dado que se acentúa la exigencia de su uso en todos los procesos sociales por sus potencialidades para la comunicación y el aprendizaje. Se considera entonces, de gran importancia, la formación de los profesores para la implementación de las TIC en la educación; dicha formación se considera uno de los requerimientos fundamentales para mejorar la calidad educativa (OIT/Unesco, 1994).

Al respecto, las estrategias de formación del profesor se entienden como "acciones educativas en el ejercicio de la profesión dirigidas a potenciar el desarrollo profesional del docente, es decir a lograr su autodeterminación en el desempeño profesional" (González y González, 2007, p. 9). Para lograrlo, entonces, resulta necesario promover en los procesos de formación: la reflexión crítica, el diálogo, la participación y el compromiso; además, "las estrategias de formación docente deben ser flexibles y contextualizadas, integrar la teoría y la práctica profesional y propiciar la reflexión crítica y comprometida del profesorado con su desempeño profesional. Deben ser útiles y prácticas" (Ibíd, p. 12). Al mismo tiempo, faciliar el intercambio de conocimientos y experiencias entre los profesores.

Algunas de las estrategias pedagógicas para la formación de las TIC se basan en:

a) la teoría de aprender a aprender,

b) la experiencia y los saberes previos de los profesores,

c) la reflexión de la práctica docente,

d) la configuración de redes de aprendizaje y el trabajo colaborativo,

e) la flexibilidad,

f) actitud y aptitud intercultural.

${ }^{6}$ En adelante se denominarán TIC a las tecnologías de la información y la comunicación. 
De acuerdo con Pernalete (2012), la formación de los profesores ha de basarse en el constructivismo social a través del enfoque dialógico e interactivo, la construcción intencional de un diálogo didáctico, cooperativo y la promoción de espacios para la reflexión. Desde este enfoque, los principios por promover son la continuidad de la formación, la integración y la diversidad, el aprendizaje autónomo y el aprender a aprender. Asimismo, Fandos, Jiménez y González (2002) formulan la importancia del trabajo en red a partir de estudiar y reflexionar acerca de la inserción de las TIC como medio didáctico con el propósito de fomentar el aprendizaje significativo. Para esto es importante tener en cuenta aspectos como: la planificación a partir de las experiencias previas y su participación en el diseño didáctico; la estructuración del contenido y el aprovechamiento de la diversidad de los materiales; el análisis de las relaciones de comunicación establecidas con las TIC y el fomento de la autonomía y la independencia.

En la revisión documental varios investigadores formulan la necesidad de formar a los profesores para el uso de las TIC, en especial, para aprovechar los recursos de internet, entre otros Anchundia y Parrales (2009); Ramírez (2012); Boza, Tirado y Guzmán (2010); Ricardo, Borjas, Velásquez, Colmenares y Serje (2013); Ortiz (2009); Trujillo (2009); Toloza, Barletta y Moreno (2013); Pozuelo (2014) y Merodo, Simón y García (2012). Éstos, en sus análisis, privilegian la figura del profesor como motor para el uso y la integración de las TIC en los currículos educativos.

Ahora bien, con respecto a las competencias de los profesores en TIC se han realizado diversas investigaciones, entre otras, las de Hennig (2013) y Suárez, Almerich, Díaz-García y Fernández-Piqueras (2012). Algunas, como las de Correa y de Pablos (2009) y Gutiérrez, (2010), plantean que ha primado más lo económico que lo innovador; las tecnologías del mañana se han utilizado para administrar el currículum del pasado. Se configuran así voces de alerta para no sobrevalorar el potencial de la TIC. Sin embargo, no emergen estudios relacionados con la normatividad de la formación.

De acuerdo con este contexto, surge los siguientes interrogantes: ¿Cuál es la normatividad del ámbito internacional y nacional acerca de la formación de profesores en TIC? ¿Cuáles han sido las estrategias promovidas por el Estado colombiano para la formación de docentes en el uso de las TIC?

Encontrar respuestas a los interrogantes formulados permite establecer un parámetro de comparación entre lo ideal de la formación de profesores en TIC, expresadas en las normas y lineamientos de poítica y las estrategias concretas realizadas con el propósito materializarlas. De igual manera, posibilita un análisis de los aspectos teóricos y prácticos implementados en la aplicación de la normatividad y la presentación de una visión de conjunto de los lineamientos de la normatividad y de las estrategias de formación de profesores llevadas a cabo para aplicarlas. El objetivo de este trabajo consiste en analizar las normas y estrategias implementadas para la formación de profesores en el uso de las TIC. Para su realización se efectuó una revisión documental de la normatividad de índole internacional y nacional acerca de la formación de profesores para el uso de las TIC y sobre las estrategias implementadas para lograrlo en Colombia. Se desarrollaron dos fases, la heurística y la hermenéutica en la que se analizó e interpretó la información sistematizada en una tabla dinámica de Excel; de acuerdo con las categorías (temáticas, objetivos, estrategias, metodología, instituciones encargadas de ofertar la formación) se realizó un balance de la aplicabilidad de dichas normas.

Los documentos formulados en relación con las políticas plantean objetivos para:
a) el acceso a las TIC mediante la dotación e infraestructura,
b) la formación pedagógica de los profesores para uso de las TIC,

c) la conformación de redes de conocimiento. 
El artículo se estructuró en los siguientes apartados:

a) metodología,

b) resultados y discusión: lineamientos de política formación TIC.

Ámbito Internacional y nacional; estrategias del Estado para la formación de profesores en el uso de las TIC,

c) conclusiones.

\section{Metodología}

La metodología utilizada para la realización del presente trabajo fue de carácter documental, con enfoque heurístico y hermenéutico. Para resolver los cuestionamientos formulados se realizó una revisión documental de diversos documentos donde se formulan políticas o lineamientos de política. En esta perspectiva, se revisaron once documentos, cinco del orden internacional:

- Informe del Consejo Económico y Social

- Declaración del Milenio

- Plan de acción de la Cumbre Mundial sobre la Sociedad de la Información

- Estándares UNESCO de Competencias en TIC para Docentes (ECD-TIC)

- Conferencia Internacional "Impacto de las TIC en Educación" en América Latina y el Caribe.

En cuanto al ámbito nacional, se analizaron seis documentos:

- Plan Nacional Decenal de Educación 2006 -2016. Lineamientos en TIC. Pacto social por la Educación, Plan Nacional de Desarrollo Educativo 20062010

- Asamblea Nacional por la Educación. Renovación pedagógica desde y uso de las TIC en la educación; Indicadores TIC para educación en Colombia; Revolución Educativa. Colombia Aprende. Plan Sectorial (2006-20010)

- Programa nacional de uso de medios y TIC; Apropiación de TIC en el desarrollo profesional docente (Ruta de Apropiación de TIC en el Desarrollo Profesional Docente); Plan Sectorial de Educación "Educación de Calidad- "El camino para la prosperidad" (2010-2014)

- Competencias TIC para el Desarrollo Profesional Docente.

El cuanto al proceso metodológico, se efectuó en dos fases: en primer lugar se desarrolló la fase heurística en la que se buscaron en diversas bases de datos, los documentos relacionados con la política y las estrategias de formación en TIC. Las categorías de búsqueda fueron:

a) normatividad sobre formación en TIC en lo internacional y nacional,

b) estrategias de formación en TIC realizadas en Colombia y consultados en el Portal

Colombia Aprende (MEN, 2015).

Luego, se sistematizó la información en una tabla dinámica realizada en el programa Excel; allí se registró el contenido de las siguientes categorías: temáticas, objetivos, estrategias, metodología, instituciones encargadas de ofertar la formación. En segundo lugar, en la fase hermenéutica, se realizó la descripción y análisis de las anteriores categorías mediante la técnica de análisis de contenido. Se buscó entonces identificar e interpretar las principales características internas de los documentos (contenido, principios, propósitos, estrategias metodológicas, resultados) y relaciones para presentar un balance de las políticas de formación y de los principales logros y requerimientos de las estrategias de formación.

\section{Resultados y discusión}

En coherencia con los interrogantes formulados, se presentan los principales aspectos de los lineamientos de política en relación con la formación en TIC y las principales características de las estrategias realizadas para lograrlos.

- Lineamientos de política internacional y nacional, formación en TIC. Los principales lineamientos de política 
referentes a la formación en TIC se establecen para propender alrededor de tres ejes importantes: el acceso a las TIC, la formación de profesores y la configuración de redes. Los documentos analizados plantean diversos objetivos en relación con:

a) el acceso a las TIC, específicamente, todo lo relacionado a la dotación e infraestructura; de ahí el énfasis en la dotación de herramientas tecnológicas y su cobertura;

b) la formación pedagógica de los profesores para su apropiación y adecuado empleo;

c) la conformación de redes de conocimiento, impulsar su desarrollo e integrarlas plenamente y de manera provechosa en la economía mundial.

\section{Infraestructura}

Referente a la infraestructura de las TIC, se plantea la necesidad de velar porque todos puedan aprovechar los beneficios de las TIC (Declaración del Milenio, ONU, 2000). Asimismo, el Plan de acción de la Cumbre Mundial sobre la Sociedad de la Información (2003) proclama que la infraestructura de las TIC es básica en la sociedad de la información; al igual que la inclusión digital para todos mediante la formación universal, el acceso sostenible, ubicuo y asequible a las TIC.

En consonancia, el Informe del Consejo Económico y Social (ONU, 2000) también propone desarrollar la infraestructura básica para implementar las TIC en todas las instituciones públicas y promover el acceso de todos a las TIC. Y de otra parte, formular políticas adecuadas para promover las inversiones en TIC y establecer el marco jurídico y regulador para su integración $\mathrm{y}$, generar, desarrollar y mejorar los contenidos locales especialmente, en idiomas locales. De igual manera, en el Plan de acción de la Cumbre Mundial sobre la Sociedad de la Información (2003) se proclamó que la infraestructura de las TIC es básica en la sociedad de la información, al igual que la inclusión digital para todos mediante la capacitación universal, el acceso sostenible, ubicuo y asequible a las TIC. Formula que un objetivo, según las circunstancias de cada país, consiste en adaptar todos los programas de estudio de los niveles educativos al cumplimiento de las metas de la sociedad de la información.

Tal como se observa, en los discursos de política, la primera preocupación fue impulsar todas las acciones relativas a la dotación de la infraestructura tecnológica a fin de garantizar el acceso de la población y garantizar su inclusión en todos los niveles educativos. Estos postulados se explican por el interés para sustentar calidad y competitividad, visión fundamentada en el utilitarismo económico. Por ejemplo, en Colombia, el Plan Nacional de Desarrollo Educativo 20062010 (MEN, 2007), enfatiza el uso de las TIC como uno de los programas estratégicos del Estado para mejorar la calidad y competitividad. Como lo indican los documentos analizados la primera preocupación fue la dotación de recursos tecnológicos y su infraestructura; de ahí, una posible desviación de la integración de las TIC en el ámbito educativo al tener la concepción de asumirlas como el equipamiento tecnológico, desde una sola perspectiva y no con una mirada integradora.

Además de la utilización de las TIC, la Unesco (2008) plantea trabajar en los siguientes componentes: práctica y formación profesional de los profesores, política educativa, pedagogía, organización y administración de la institución educativa, al igual que su inclusión en el plan de estudios (currículo), las formas de evaluación y la investigación.

\section{Formación de profesores}

Éste componente no sólo expresa el propósito por obtener la competitividad, pues también se enuncian propósitos relacionados con la calidad y equidad de la educación; por tanto, se plantea la relevancia de la formación de los profesores como un factor fundamental para lograrlas. La perspectiva es abordar las TIC como mediadoras de la generación de 
conocimiento y desarrollo del pensamiento crítico. El citado Informe del Consejo Económico y Social (ONU, 2000) propone hacer inversiones necesarias para el desarrollo de los recursos humanos y reforzar las instituciones y las redes para la producción, adquisición, absorción y difusión de productos basados en los conocimientos. Asimismo, el Plan de acción de la Cumbre Mundial sobre la Sociedad de la Información (2003), que formula la creación de capacidades mediante la preparación de profesores para afianzar el concepto de formación continua, analizar $\mathrm{y}$ tratar la información de manera creativa e innovadora y, en esa medida, formar una masa crítica altamente calificada de profesionales y expertos en las TIC.

La preocupación esencial es la eficacia de la enseñanza para "crear sociedades del conocimiento [que sean] inclusivas mediante la comunicación y la información (Unesco, 2008 , p. 3), en el marco de la educación de calidad para todos. En esta dinámica, hay una complementación, las TIC y la formación de profesores para su uso no sólo se consideran el apalancamiento para la competencia en la producción nacional; también, se perciben como elementos importantes para lograr calidad y equidad. Este aspecto, se puede analizar como un avance de los discursos, aunque no se desplaza la competitividad, entonces, se articula con otras grandes metas del Estado. En este sentido, se relaciona la formación en TIC con la producción de conocimiento. La estrategia específica de formación propuesta es realizarla por niveles para desarrollar los siguientes enfoques:

a) nociones básicas de TIC,

b) profundización del conocimiento, $\mathrm{y}$

c) generación de conocimiento.

Acorde con los enfoques de los niveles de formación, los objetivos son:

a. la integración de competencias en TIC en los currículos (enfoque de nociones básicas de TIC) a través de la obtención, por parte de los profesores, de competencias básicas en TIC para que "estén en capacidad de usar las TIC para gestionar datos de la clase y apoyar su propio desarrollo profesional" (Unesco, 2008, p. 12); además de seleccionar y utilizar métodos educativos apropiados para diferentes usos, prácticas y contenidos de internet.

b. La resolución de problemas complejos y reales (enfoque de profundización del conocimiento) que comprende la capacidad para gestionar información, crear y supervisar proyectos de clase, contactar expertos y utilizar redes para colaborar con otros profesores, acceder a información y "contribuir a su propio desarrollo profesional" (Unesco, 2008, p. 12).

c. Promover la innovación y la producción de conocimiento (enfoque de generación de conocimiento). Los profesores podrán crear comunidades de conocimiento basadas "en la innovación y en el aprendizaje permanente, enriquecidos por las TIC" (Unesco, 2008, p. 14), mediante el diseño de recursos y ambientes de aprendizaje con la utilización de las TIC para apoyar el desarrollo de generación de conocimiento $\mathrm{y}$ de habilidades de pensamiento crítico y reflexivo.

Las formulaciones de la Unesco, entonces, se consideran un avance porque la propuesta es integrar, por una parte, los conocimientos técnicos de índole instrumental y utilitarista $\mathrm{y}$, por otro lado, el desarrollo del pensamiento crítico y creativo mediante la realización de proyectos pilotos en las instituciones educativas. Por ello, "[...] uno de los mayores desafíos es desarrollar competencias en el ámbito del uso de las tecnologías vinculadas a lo pedagógico; y a la gestión conjunta $\mathrm{y}$ coherente de ambas dimensiones" (Unesco, 2010 , p. 34). La apuesta entonces consiste en trabajar en modelos pedagógicos adecuados a los distintos tipos de escuelas. De ahí el reto, la fundamentación del modelo pedagógico de acuerdo al tipo de sociedad.

En Colombia se siguieron las directrices de la Unesco y se expidió la regulación correspondiente, acorde con los parámetros formulados en el ámbito internacional. En la Ley 115 de 1994, se determina como uno de los factores de eficacia educativa la 
formación de los profesores, puesto que “[...] el Estado deberá atender en forma permanente los factores que favorecen la calidad y el mejoramiento de la educación; especialmente velará por la cualificación y formación de los educadores, la promoción docente..." (Ley 115, Art. 4). Esto se ratifica en el Art. 109, de la misma Ley donde se especifican las finalidades de la formación con fundamento en la pedagogía y la investigación.

En el "Plan Nacional Decenal de Educación 2006 -2016. Lineamientos en TIC. Pacto social por la Educación" (Ministerio de Educación Nacional (MEN), 2006), un macro objetivo es la formación inicial y permanente de docentes en el uso de las TIC para lo cual, se pretende fortalecer la formación pedagógica en las escuelas normales y las universidades, para formar un estudiante activo mediante la investigación educativa y el uso de las TIC. Así, se instituye el diseño de los currículos con base en la investigación y el uso transversal de las TIC. Como efecto, se incentiva la innovación educativa y pedagógica junto con la implementación de experiencias significativas y redes colaborativas virtuales; relaciones que contribuirán al desarrollo del aprendizaje autónomo, al pensamiento crítico y al trabajo colaborativo. Desde estos lineamientos, la exigencia es emplear las TIC con un enfoque pedagógico que supere lo tradicional y privilegie el aprendizaje significativo en la perspectiva de superar su formación instrumental.

Para la materialización de este ideario, en el Plan Nacional de Desarrollo Educativo 20062010 (MEN, 2007) se propone el fomento a la generación de proyectos de investigación en el marco de la Red Nacional Académica de Tecnología Avanzada (RENATA) ${ }^{7}$, como herramienta de apoyo a los procesos investigativos. Para los profesores universitarios, presenta la Vitrina eLearning de programas de formación en uso de TIC y desarrollo de soluciones de formación flexible (basada en $\mathrm{OA})^{8}$. Asimismo, en el documento "Asamblea Nacional por la Educación. Renovación pedagógica desde y uso de las TIC en la educación" (MEN, 2007), se hace hincapié en la renovación pedagógica con base en el aprendizaje del estudiante como sujeto activo. Así, se reitera la formación permanente del profesor para el uso apropiado y responsable de las TIC mediante la integración de la investigación y el uso crítico y reflexivo de éstas. Esta perspectiva de apropiación de las TIC a través de la investigación se constituye en un reto para lograr la formación de los profesores. Igualmente, se propone incorporarlas como eje transversal a fin de fortalecer los procesos de enseñanza y aprendizaje en todos los niveles educativos y para afianzar la ciudadanía y la competitividad.

En coherencia con lo planteado por la Unesco (2008), el MEN (2013) plantea como competencias básicas de los profesores para el uso de las TIC, las siguientes:

a. tecnológica

b. investigativa

c. gestión pedagógica, directiva,

administrativa y comunitaria

d. comunicativa

e. pedagógica.

Con estos criterios en mente, entonces, se pretendetransformar los saberesy la generación de nuevos conocimientos que aporten al desarrollo de los pueblos. Asimismo, formula

\footnotetext{
7 “RENATA es la red nacional de investigación y educación de Colombia que conecta, articula e integra a los actores del Sistema Nacional de Ciencia Tecnología e Innovación (SNCTI) entre sí y con el mundo, a través del suministro de servicios, herramientas e infraestructura tecnológica para contribuir al mejoramiento del nivel de productividad, efectividad y competitividad de la producción científica y académica del país". RENATA, 2010).

${ }^{8} \mathrm{OA}$, sigla de objeto de aprendizaje; “... conjunto de recursos digitales, auto contenible y reutilizable, con un propósito educativo y constituido por al menos tres componentes internos: Contenidos, actividades de aprendizaje y elementos de contextualización. El objeto de aprendizaje debe tener una estructura de información externa (metadatos) que facilite su almacenamiento, identificación y recuperación” (MEN, 2006)
} 
su estructura en tres niveles o momentos: exploración, integración e innovación.

De otra parte, los propósitos de la inclusión de las TIC en la educación son:

a. apoyo transversal para mejorar la competitividad del país y potenciar el crecimiento de la productividad de los sectores económicos

b. apoyo a los nuevos sectores económicos basados en la innovación

c. herramienta de buen gobierno (fortalecimiento institucional, transparencia, rendición de cuentas, gobierno en línea, entre otros).

(MEN, 2013, p.15).

No obstante, estas intenciones y proyecciones de las TIC en la producción de conocimiento, básicamente se conciben, como mediadoras o puentes para que los estudiantes sean ciudadanos informados, responsables $y$ capaces de contribuir a la sociedad, pero ante todo, trabajadores colaboradores, actualizados y en aprendizaje permanente a través de las TIC, competencias requeridas para la eficacia y eficiencia de la producción económica. Uno de los programas estratégicos para la competitividad es el uso de las TIC para utilizarlas de manera eficiente y soporte importante para el desempeño exitoso en cualquier entorno laboral, que asimismo requiere:

Como ejes de trabajo: a) construcción de una infraestructura de calidad y desarrollo de contenidos, b) definición de estándares y formación de estudiantes y docentes para el uso adecuado y la apropiación de las nuevas tecnologías en la educación y, c) consolidación de comunidades y redes educativas virtuales que aprovechen las ventajas que ofrecen las TIC y generen nuevos conocimientos a partir de su explotación (MEN, 2008, p.47).

De ahí la propuesta de llevar a cabo una adaptación curricular con su inclusión y la certificación de los profesores en competencias digitales, para lograr en la educación la innovación y la productividad, tal como se plantea en el Plan Sectorial de Educación "Educación de Calidad-El camino para la prosperidad" (2010-2014).

\section{Conformación de redes}

La intencionalidad consiste en apoyar las actividades que los países llevan a cabo con miras a aprovechar las posibilidades de las TIC, impulsar su desarrollo e integrarlas plenamente y de manera provechosa en la economía mundial con base en las redes de información y conocimientos. En consecuencia, la apropiación de las TIC en diversos ámbitos sociales fortalecerá las relaciones de mercado, propio del modelo neoliberal. En el país, en los indicadores TIC para educación en Colombia (MEN, 2007), se formula como resultados de la formación de los profesores, su participación en actividades de interacción y colaboración en la red escolar y, la realización de tráfico de uso de los contenidos y servicios de red - Portal. Para tal efecto, los profesores diseñarán portafolios de actividades de experiencias significativas de aprendizaje en las que se hace uso de TIC, aportes de renovación pedagógica Institucional y el nivel de motivación de los estudiantes frente al aprendizaje.

\section{Estrategias del Estado: formación de profesores en el uso de las TIC}

En cuanto a la estrategia de formación por niveles y por fases, el MEN ha impulsado el desarrollo de diversos tipos de cursos con el propósito de aplicar las políticas de formación de profesores en el uso de las TIC. Las fases programadas, según el MEN (2015) son:

a. fase A: inicial,

b. fase B: de profundización (estrategia de formación para los maestros)

c fase $\mathrm{C}$ : de aprendizaje en ambientes virtuales (estrategia de formación para la comunidad educativa):

Por ejemplo, A que te cojo ratón desarrolla la fase A: inicial. En la tabla 1 se indica la metodología, el contenido y la institución o entidad encargada de la formación. 
Tabla 1. Programa de formación: A que te cojo ratón

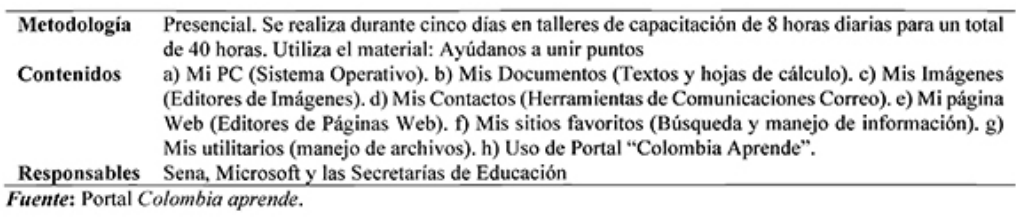

Este programa constituye el primer nivel de formación, el cual se ocupa del plano instrumental, aspecto importante y necesario; sin embargo, no considera la reflexión acerca del porqué es importante integrar el uso de las TIC a la educación, cuáles son sus relaciones con la cultura y cómo inciden en las diversas prácticas sociales. Es decir, no hay un acercamiento a las prácticas reflexivas que implique superar el acercamiento utilitarista de las TIC.

\section{TemaTICas}

Este programa fue desarrollado por el MEN y dirigido a directivos docentes (DD) de instituciones educativas, para cualificar su labor y promover procesos de mejoramiento, transformación y cambio institucional con apoyo de las TIC. Concertado a través de la conformación de equipos de gestión conformado por el rector, o más directivos, un docente, un estudiante, un padre de familia y un participante de la comunidad, que demuestren actitud positiva para el aprendizaje permanente y deseen ser actores claves para la construcción de planes institucionales de uso de TIC. En este proceso hacen un tránsito de aprendices a formadores en su propia institución, dado que sus aprendizajes y orientaciones se reflejarán luego en su desempeño. En la tabla 2 se indican las características.

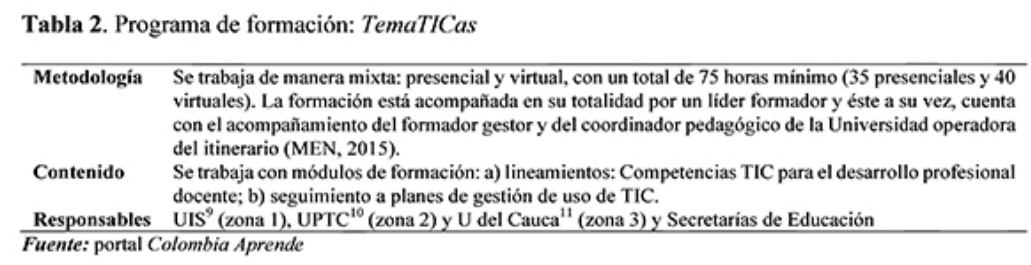

En este programa se percibe interés por la aplicabilidad del uso de las TIC y el trabajo cooperativo de varios formadores. El líder formador será el artífice de la expansión de la formación recibida en su institución. Es una estrategia de cascada, se forman lo formadores quienes a su vez, forman a otros. Esta dinámica es importante, pero se desconocen las condiciones institucionales de apoyo que faciliten la realización de esta labor.

\section{INTEL}

Este programa consiste en un modelo de formación en cascada, que cuenta con un grupo de docentes líderes formadores (senior trainers - master teachers), con un alto nivel de competencias en uso pedagógico de TIC, quienes forman a los docentes participantes (DP), con el propósito de integrar el uso pedagógico de TIC en las prácticas educativas. El programa es interesante porque promueve el aprendizaje colaborativo; no obstante, no queda claro cuál es la fundamentación pedagógica para incentivar la investigación y la apropiación cultural del uso de las TIC. En la tabla 3 se señalan algunos aspectos

\footnotetext{
${ }^{9}$ UIS: Universidad Industrial de Santander

${ }^{10}$ UPTC: Universidad Pedagógica y Tecnológica de Colombia.

${ }^{11}$ U del Cauca: Universidad del Cauca.
} 
Tabla 3. Programa de formación: INTEL

\begin{tabular}{|c|c|}
\hline Metodología & $\begin{array}{l}\text { Una variación es el programa: Intel Educar Introductorio en uso pedagógico de las TIC en el marco } \\
\text { del programa Todos a Aprender. Los actores se denominan: a) formadores, b) tutores; c) docentes } \\
\text { acompañados. Los senior trainers (ST): docentes con experiencia en formación de docentes en el } \\
\text { uso de tecnologías y se encargan de formar a los master teachers (MT). Docentes seleccionados } \\
\text { para ser formados en el programa por los ST. Su función primordial es formar a sus pares como } \\
\text { Docentes Participantes (DP), quienes a su vez, son docentes que reciben la formación en el } \\
\text { Programa. Se priorizan aquellos que desarrollan su labor en condiciones dificiles. De igual manera, } \\
\text { se busca replicar el modelo en diferentes regiones. }\end{array}$ \\
\hline Conte & $\begin{array}{l}\text { En su desarrollo se fomentan las competencias digitales y en innovación, para la cualificación de } \\
\text { la práctica pedagógicas de los profesores con ayuda de las TIC. }\end{array}$ \\
\hline sponsables & MEN en alianza con Intel \\
\hline
\end{tabular}
Fuente: Portal Colombia Aprende

\section{Entre pares}

Con este programa se forman docentes (facilitadores) que forman a otros docentes (asesores). Se continúa en la misma visión de la estrategia cascada implementada en las anteriores. Se parte del presupuesto que entre pares hay mayor interacción y colaboración. En la tabla 4 se especifica el tipo de formación.

Tabla 4. Programa de formación: Entre pares

\begin{tabular}{cl}
\hline Metodología & $\begin{array}{l}\text { Se fundamenta en la estrategia de coaching (asesoría entre colegas). Un docente comparte su saber } \\
\text { en uso pedagógico de TIC con sus pares en la institución, a través del diseño de actividades de } \\
\text { aprendizaje: (weblesson). Busca la integración de las TIC a los contenidos curriculares. }\end{array}$ \\
Contenidos & $\begin{array}{l}\text { El contenido se desarrolla en seis unidades: 1- Importancia de la formación a través de asesores } \\
\text { como estrategia eficaz para la formación docente. 2- Se profundiza en el rol del asesor y las } \\
\text { herramientas para la asesoria, se presentan técnicas de comunicación para establecer relaciones de } \\
\text { colaboración sólidas. 3- Weblesson: actividad de aprendizaje para publicar en internet. Para ello } \\
\text { deben escribir una pregunta generadora, determinar las tareas que los estudiantes realizan, vincular }\end{array}$ \\
la unidad a los objetivos del plan de estudios, establecer criterios de evaluación, elaborar \\
herramientas para el andamiaje e identificar los recursos necesarios para completar la lección. 4- \\
Aprendizaje colaborativo/cooperativo mediado por computador; viven la experiencia de navegar y \\
evaluar proyectos colaborativos de diversos portales educativos latinoamericanos. 5- Acceso, a \\
través de herramientas como el foro y el chat y a documentos que les permiten reflexionar sobre la \\
integración de la tecnología al currículo. 6- Reflexión acerca de su labor como facilitadores, en la \\
medida en que exploran recursos relacionados con la colaboración entre comunidades locales y \\
globales. \\
MEN en cooperación con Microsoft
\end{tabular}

En este programa se vincula mucho más la parte pedagógica mediante la fundamentación del trabajo cooperativo con los aspectos utilitarios de las TIC. Hay un contacto inicial con proyectos de investigación en la perspectiva de servir como modelos de la incorporación pedagógica de las TIC en los ambientes de aprendizaje; pero no se profundiza en el componente investigativo.

\section{CREA TIC}

Es un componente de la formación de docentes a través del fortalecimiento de sus habilidades con uso de TIC, el fomento de investigación y el desarrollo de estrategias innovadoras a través del uso y el desarrollo de contenidos digitales educativos. El programa es ofrecido por el proyecto: "Construyendo capacidades en uso de las TIC para innovar la educación". En la tabla 5 se indican sus aspectos.

Tabla 5. Programa de formación: CREA TIC

\begin{tabular}{ll}
\hline Metodología & $\begin{array}{l}\text { Su postulado es inspirar, crear y diseñar con TIC en el aula de clases y el desarrollo de } \\
\text { competencias de: a) diseño, tecnológica, pedagógica, comunicación e investigación. Es un } \\
\text { programa b-learning conformado por dos cursos virtuales (primera y cuarta semana) y talleres } \\
\text { presenciales (segunda y tercera semana) organizados en cuatro fases. Los cursos virtuales son } \\
\text { autorregulados y se aplica el aprendizaje colaborativo. Se cran comunidades de práctica. } \\
\text { Básicamente se busca: planear, diseñar y evaluar unidades didácticas. También la realización de } \\
\text { investigación educativa con el apoyo de las TIC. }\end{array}$ \\
Se ofrecen dos cursos: - Virtual I: Innovación en el aula de clase con el uso educativo de TIC. \\
Prácticas: a) Autoevaluación habilidades en TIC. b) Políticas educativas para el uso de TIC en el \\
aula. c) Importancia de la planeación de unidades didácticas con TIC. d) Estrategia de coaching \\
(acompañamiento y orientación) para docentes. e) Diseño de estrategias para el uso pedagógico de \\
tecnologias emergentes. g) Plan de coaching y acompañamiento con los docentes. - Virtual 2: \\
Ambientes de aprendizaje orientados al futuro. Se trata de aplicar lo aprendido. \\
En los talleres, se trabaja en: a) Implementación de unidades didácticas, b) Investigación de las \\
prácticas educativas (Modelos de investigación y de análisis de datos c) Bitácora.
\end{tabular}


En este programa hay mayor correspondencia entre lo estipulado en los discursos de la normatividad acerca de la formación de profesores en uso de las TIC y, en particular, en la fundamentación pedagógica e investigativa que contribuya a su empleo innovador. No obstante, a pesar de estos avances, no hay una reflexión acerca de la perspectiva cultural para su incorporación en los entornos educativos.

Por otra parte, hay dos programas preocupados básicamente por la dotación de infraestructura tecnológica en las instituciones educativas que a la par han dado formación instrumental para el uso de las TIC. Estos programas han constituido el primer acercamiento a las TIC por parte de los profesores y de la comunidad educativa en general. Conjugan los dos grandes objetivos de las políticas, la infraestructura y la formación pero con una cobertura mayor, la comunidad educativa. En la tabla 6, se indican algunas de sus características.

Tabla 6. Programas de formación: Vive Digital y Computadores para Educar

\begin{tabular}{|c|c|c|}
\hline Programa & Vive Digital (MinTIC) & Computadores para Educar \\
\hline Metodología & $\begin{array}{l}\text { Busca que el pais dé un gran salto } \\
\text { tecnológico mediante la masificación de } \\
\text { internet y el desarrollo del ecosistema } \\
\text { digital nacional para la generación de } \\
\text { empleo y la reducción de la pobreza. }\end{array}$ & $\begin{array}{l}\text { El programa consiste en la donación de equipos de } \\
\text { cómputo por parte de empresas públicas y privadas } \\
\text { a escuelas y colegios públicos del pais }\end{array}$ \\
\hline Contenidos & $\begin{array}{l}\text { Buscar otorgar un papel importante a la } \\
\text { promoción de actividades de investigación } \\
\text { y desarrollo en el ámbito TIC }\end{array}$ & $\begin{array}{l}\text { La formación se considera un proceso intencional y } \\
\text { planeado, que se sustenta en la teoria y en la } \\
\text { reflexión, y que responde a las necesidades de } \\
\text { transformación de las prácticas a través de la } \\
\text { vinculación de las TIC como recurso fundamental } \\
\text { para el aprendizaje (MEN, 2015). }\end{array}$ \\
\hline Responsables & $\begin{array}{l}\text { El Ministerio de Educación, las } \\
\text { universidades, el Sena, departamentos y } \\
\text { municipios. }\end{array}$ & $\begin{array}{l}\text { El Ministerio de Comunicaciones, el Ministerio } \\
\text { Educación, el Sena, Telecom y el Gobierno de } \\
\text { Canadá }\end{array}$ \\
\hline
\end{tabular}

Tal como se indicó, la perspectiva de estos dos programas es el acceso y la equidad a través de la cobertura brindada mediante la dotación y equipamiento de la infraestructura y su manejo tecnológico, lo cual constituye el primer nivel de la formación promovida en los diversos discursos de las políticas.

Dado el reconocimiento del papel fundamental desempeñado por el profesor para realizar el cambio en el campo educativo, es indiscutible que en el país se han hecho esfuerzos por formular documentos de lineamientos de políticas y estrategias de formación en el uso de las TIC. Si bien es cierto, se basan en lo formulado internacionalmente, el MEN ha impreso su propio carisma al promover la fundamentación pedagógica, comunicativa e investigativa.

Ahora bien, se desconoce si efectivamente todos los profesores han realizado la formación en todos los niveles, tal como se propone y cuál ha sido el grado de transformación percibido en el currículo y en el mejoramiento de las prácticas de enseñanza y aprendizaje. De acuerdo con las estrategias analizadas, aún se adolece de la suficiente fundamentación pedagógica e investigativa para contribuir a la apropiación crítica y creativa de las TIC en los ambientes de aprendizaje.

En parte, esto se puede explicar porque los encargados de dirigir dicha formación, en su mayoría, han sido organismos ajenos a la Universidad $^{12}$, institución que tiene como misión privilegiar la investigación y la fundamentación pedagógica de las prácticas educativas.

De igual manera, aún no es fuerte el análisis de las relaciones de poder inmersa en los ambientes de aprendizaje mediados por las TIC; en éstos se establecen redes de significaciones mediante las prácticas culturales en el entorno de lo social y lo político, relacionadas con las

\footnotetext{
${ }^{12}$ Sólo en el programa TemaTICas, algunas universidades son las responsables (UIS, UPTC y U del Cauca). En Vive Digital, se expresa que las universidades participan pero no se especifica cuáles son.
} 
representaciones y prácticas del poder. Por tanto, es importante incluir en los procesos de formación reflexiones de carácter cultural, acerca del tipo de sociedad en la que se aspira brindar la formación y el tipo de hombre que se quiere formar con la mediación de las TIC. Con el empleo de las TIC no hay una neutralidad cultural, pues según la opción se privilegia determinada cultura: "[...] los ordenadores dan soporte a diferentes estilos y culturas ya que podemos aproximarnos a los mismos de diferentes maneras" (Turkle, 1997, p. 44).

Por otra parte, se requiere mayor fundamentación de lo comunicativo para su apropiación. Los ambientes de aprendizaje mediados por las TIC, ante todo son espacios de intercambio comunicativo entre colectivos con un interés común; promueven básicamente el aprendizaje interactivo y las condiciones para establecer relaciones comunicativas entre los diversos actores; por tanto, no solamente han de circular contenidos. Al respecto, Piscitelli propone la necesidad de la apropiación de las TIC como una forma de: "[...] competencia en el lenguaje, como medio de diseño de nuevas prácticas para hacernos cargo mejor de nuestros intereses en el mundo" (Piscitelli, 2002, p. 62); es decir, plantea asumir con visión crítica y creativa las prácticas y herramientas a partir de las conversaciones e intercambios que surgen en la vida cotidiana, de tal manera que se incremente la capacidad de comunicación humana: las relaciones horizontales y no el aumento del poder. Se trata de reflexionar sobre lo nuestro, nuestra cultura para acceder desde allí a los mundos virtuales y determinar los modos de convivencia que se desean alcanzar. De ahí que se aboga por la identidad cultural, las memorias colectivas y la acción práctica. Es una visión de tiempos mestizos e híbridos.

\section{Conclusiones}

En los lineamientos de políticas para la inserción de las TIC en los ambientes educativos se proponen básicamente tres objetivos o ejes:

a. Lograr el acceso y equidad a través la dotación de la infraestructura de las TIC.

b. Brindar calidad y competitividad de la educación mediante la formación de los profesores para la apropiación pedagógica de las TIC

c. El trabajo en red para fomentar la producción e intercambio de conocimiento, con la mediación de las TIC.

Han sido varias las estrategias implementadas en Colombia para la formación de los profesores. La propuesta ha sido realizarla por niveles $y$, en varios casos, se ha trabajado en la formación en cascada. La propuesta es buscar la formación integral: tecnológica, pedagógica, comunicativa, investigativa y gestión pedagógica, directiva, administrativa y comunitaria. Sin embargo, varias prácticas de formación aún no superan la formación instrumentalista de las TIC y no se han logrado fundamentar con mayor solidez lo pedagógico, comunicativo e investigativo. Una reflexión ausente son las relaciones culturales y de poder inherentes a la inserción de las TIC en los diferentes ámbitos sociales para propender así por su apropiación crítica e innovadora en la realidad del contexto educativo.

Finalmente, se requiere participar en la consolidación del análisis de lo cultural desde la pedagogía y la investigación como tarea que todavía no asume la universidad como centro por excelencia de formación.

\section{Referencias}

Anchundia, M. \& Parrales, A. (2009). La utilización del internet por parte del docente como recurso para el desarrollo de la lectura comprensiva de los estudiantes del octavo año de Educación Básica del Colegio nacional Bahía de Manta de la parroquia de los Esteros del Cantón Manta Duran. (Tesis de grado). Facultad de Ciencias de la Educación. Universidad Laica "Eloy Alfaro" de Manabí.

Boza, A., Tirado, R. \& Guzmán, M. (2010). 
Creencias del profesorado sobre el significado de la tecnología en la enseñanza: influencia para su inserción en los centros docentes andaluces. Revista electrónica de investigación y Evaluación educativa, 16 (1), 1-24.

Carnoy, M. (2001). El impacto de la mundialización en las estrategias de reforma educativa. Revista de Educación (236-241), 101-110.

Correa, J. M. y De Pablos, J. (2009). Nuevas Tecnologías e Innovación Educativa. Revista de Psicodidáctica, 14. (1), 133-145

Fandos, M., Jiménez, J. y González, A. P. (2002). Estrategias didácticas en el uso de las Tecnologías de la Información y la Comunicación. Acción Pedagógica, 11, (1), 28-39. Recuperado de http://www. comunidadandina.org/bda/docs/VEEDU-0003.pdf

González, R.M. y González, V. (2007). Diagnóstico de necesidades y estrategias de formación docente en las universidades. Revista Iberoamericana de Educación (43/6), 1-14, Recuperado de http://www.ub.edu/ obipd/PDF\%20docs/Formaci\%C3\%B3\%20 Inicial/Educaci $\% \mathrm{C3} \% \mathrm{~B} 3 \% 20$ Universitaria/ Publicacions/Diagn \%C 3\%B3 stico\%20 de $\% 20$ necesidades $\% 20 y \% 20$ estrategias $\% 20$ de $\% 20$ formaci $\% \mathrm{C} 3 \% \mathrm{~B} 3 \mathrm{n} \% 20$ docente $\% 20$ en $\% 201$ as $\% 20$ universidades. $\% 20$ Gonz\%C3\%A1lez,R.M.\%20Gonz\%C3\%Allez,V.pdf

Gutiérrez, A. (2010). La formación de los futuros maestros y la integración de las TIC en la educación: anatomía de un desencuentro. Revista de Educación, (352).

Hennig, C. (2013). Formación de competencias docentes en TIC: Retos y desafíos. Ponencia presentada en VirtualEduca. Formación de competencias docentes.

Krüger, K. (2006). El concepto de sociedad del conocimiento. Revista Bibliográfica de
Geografía y Ciencias Sociales. 11, (683).

Ley General de Educación. (Ley 115 de 1994). Congresode la República de Colombia. Bogotá. Recuperado de http://www.secretariasenado. gov.co/senado/basedoc/ley_0115_1994.html

Ministerio de Educación Nacional (MEN) (2006). Plan Nacional Decenal de Educación 2006 -2016. Lineamientos en TIC. Pacto social por la Educación. Recuperado de http://www.plandecenal.edu.co/html/1726/ articles-129419_archivo.pdf

(2007).Plan Nacional de

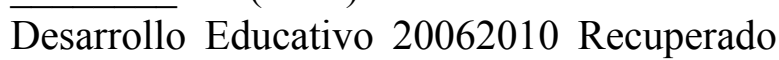
de http://www.mineducacion.gov.co/1621/ articles-119059_archivo_pdf1.pdf

(2007). Asamblea Nacional por la Educación. Renovación pedagógica desde y uso de las TIC en la educación. Recuperado de http://www.plandecenal.edu.co/html/1726/ articles-166057_TICS.pdf

(2007). Indicadores TIC para educación en Colombia. Recuperado de https://www.itu.int/ITU-D/ict/events/ dominicanrep08/material/Colombia.pdf

(2008). Revolución Educativa. Colombia Aprende. Plan Sectorial (200620010) Programa nacional de uso de medios y TIC. Recuperado de http:// www.colombiaaprende.edu.co/html/ investigadores/1609/articles-73383_ documento_pdf.pdf

(2008). Apropiación de TIC en el desarrollo profesional docente (Ruta de Apropiación de TIC en el Desarrollo Profesional Docente. Recuperado de http:// es.slideshare.net/wilcox1/ruta-apropiacin-detic-en-el-desarrollo-profesional-docente-paraeducacin-superior-men

(2010). Plan Sectorial de Educación "Educación de Calidad- "El camino para la prosperidad" (2010-2014). 
Recuperado de http://planipolis.iiep.unesco. org/upload/Colombia/Colombia_Plan sectorial_2010-2014.pdf

(2013). Competencias Tic para el desarrollo profesional docente. Recuperado de http://www.colombiaaprende.edu.co/html/ micrositios/1752/articles-318264_recurso_ tic.pdf

(2015). Colombia aprende. Recuperado de http://www.colombiaaprende. edu.co/html/home/1592/w3-channel.html

Merodo, A., Simón, J. y García, M. X. (2012).La incorporación de las TIC en la formación inicial docente. Recuperado de http://craig.com.ar/biblioteca/La $\% 20$ incorporaci $\% \mathrm{~F} 3 \mathrm{n} \% 20 \mathrm{de} \% 201 \mathrm{as} \% 20 \mathrm{TIC} \% 20$ en $\% 201 \mathrm{a} \% 20$ Formaci $\%$ F3n $\% 20$ Inicial $\% 20$ Docente \% 20-\%20Fundaci \%F 3n\%20 Evoluci\%F3n.pdf

ONU. Asamblea General (2000). Informe del Consejo Económico y Social correspondiente a 2000. Recuperado de http://www.itu.int/ wsis/docs/background/resolutions/55-3-es.pdf (2000). Declaración del Milenio.

ONU ITU (2003). El Plan de acción de la Cumbre Mundial sobre la Sociedad de la Información. Recuperado de https:// www.itu.int/wsis/documents/doc_multi. asp?lang=es\&id $=1160 \% 7 \mathrm{C} 0$

Ortiz, M. (2009). Valoración y gusto por la lectura en alumnos de $3^{\circ}$ y $4^{\circ}$ básico. (Tesis de grado). Facultad de Educación. Universidad Academia de Humanismo Cristiano de Chile.

Pérez, C. (1996). Las normas en el currículum escolar. Revista española de Pedagogía. (205), 535-556

Pernalete, D. (2012). Formación docente en estrategias didácticas con TICs. Revista Innovación Educativa, 12, (58), 119-132.
Piscitelli, A. (2002). Ciberculturas 2.0. En: La era de las máquinas inteligentes. Buenos Aires: Paidós.

Pozuelo, J. (2014) ¿Y si enseñamos de otra manera? Competencias digitales para el cambio metodológico. Caracciolos, 2 (1)

Ramírez, E. (2012). La incorporación de la cultura digital en las prácticas de lectura de los estudiantes de bachillerato de la UNAM. Revista Investigación Bibliotecológica, 26 (56), 43-69.

Ricardo, C., Borjas, M., Velásquez, I., Colmenares, J. \& Serje, A. (2013). Caracterización de la Integración de las TIC en los currículos escolares de las instituciones educativas en la ciudad de Barranquilla. Revista del Instituto de Estudios en Educación Universidad del Norte, Zona próxima, (18), $32-45$.

Suárez, J.M. Almerich, G., Díaz-García, I. y Fernández-Piqueras, R. (2012). Competencias del profesorado en las TIC. Influencia de factores personales y contextuales. Univ. Psychol, 11 (1), 293-309

Toloza, H., Barletta, N. \& Moreno, F. (2013). Una experiencia de acompañamiento en el proceso de enseñanza y aprendizaje de la lectura y la escritura en educación media. Revista del Instituto de estudios en Educación Universidad del Norte, (19), 39-55.

Trujillo, F. (2009). Prácticas de lectura literaria en aulas de secundaria. Textos en contexto, Revista lectura y vida, 29-39.

Turkle, S. (1997). La vida en la pantalla. La construcción de la identidad en la era de Internet. Barcelona: Paidós.

Unesco (2008). Estándares UNESCO de Competencia en TIC para Docentes: (ECDTIC). Recuperado de http://www.oei.es/tic/ UNESCOEstandaresDocentes.pdf 
(2010). Conferencia Internacional "Impacto de las TIC en Educación" en América Latina y el Caribe. Recuperado de http://unesdoc.unesco.org/ images/0019/001905/190555s.pdf 